\title{
FINITE ELEMENT ANALYSIS FOR UNSTEADY MHD HEAT AND MASS TRANSFER FREE CONVECTION FLOW OF POLAR FLUIDS PAST A VERTICAL MOVING POROUS PLATE IN A POROUS MEDIUM WITH HEAT GENERATION AND THERMAL DIFFUSION
}

\section{Bala Siddulu Malga ${ }^{1}$ and Naikoti Kishan ${ }^{2}$}

${ }^{1}$ Department of Mathematics, GITAM University, Hyderabad, Telangana, India, 502329, Email:balumalga@gmail.com

${ }^{2}$ Department of Mathematics, University College of Science, Osmania University, Hyderabad, Telangana, India-500007 Email: kishan_n@ rediffmail.com

\begin{abstract}
:
The unsteady two-dimensional magneto-hydrodynamic heat and mass transfer free convection flow of an incompressible viscous electrically conducting polar fluid through a porous medium past a semi-infinite vertical porous moving plate in the presence of a transverse magnetic field with thermal diffusion and heat generation is considered. The plate moves with a constant velocity in the longitudinal direction and the free stream velocity follows an exponentially increasing or decreasing. A uniform magnetic field acts perpendicularly to the porous surface which absorbs the polar fluid with a suction velocity varying with time. The equations of conservation of mass, momentum, energy and concentration which govern the case study of heat and mass transfer flow have been obtained. The equations have been solved numerically by Galerkin finite element method. The effect of various flow parameters are presented graphically. Representative results for velocity profiles, temperature profiles and concentration profiles are obtained for several values of pertinent parameters which are of physical and engineering interest.
\end{abstract}

Keywords:MHD; Free convection, porous medium, heat and mass transfer, thermal diffusion, finite element method

\section{NOMENCLATURE}

A suction velocity parameter

$B_{0} \quad$ magnetic flux density

C concentration of the fluid within the boundary layer

$\mathrm{Cp} \quad$ specific heat at constant pressure

Gr Grashof number

Gc modified Grashof number

$g \quad$ acceleration due to gravity

$K \quad$ permeability parameter

$k \quad$ thermal conductivity

$M \quad$ magnetic parameter

$N$ dimensionless material parameter

$n$ dimensionless exponential index

$\mathrm{Pr} \quad$ Prandtl number

$T$ temperature

$t$ dimensionless time

$U_{0} \quad$ scale of free stream velocity

$U, V \quad \begin{aligned} & \text { component of velocit } \\ & \text { the plate, respectively }\end{aligned}$

\section{Greek symbols}

$B$ dimensionless viscosity ratio

$\beta_{f}$ coefficient of volumetric expansion of $f$ the working fluid

$\beta_{c}$ coefficient of concentration expansion

$\gamma \quad$ spin-gradient viscosity

$\ddot{\varepsilon} \quad$ scalar constant $(<<1)$

$\sigma$ electrical conductivity

$\rho$ fluid density

$\Lambda$ coefficient of gyro-viscosity

$\mu$ fluid dynamic viscosity

$v$ fluid kinematic viscosity

$v_{r}$ fluid kinematic rotational viscosity

$\theta$ dimensionless temperature

$\omega$ angular velocity vector

$\varnothing$ dimensionless concentration

\section{Superscripts}

differentiation with respect to $y$

* dimensional properties 


$x, y \quad \begin{aligned} & \text { distances along and perpendicular to the plate } \\ & \text { respectively }\end{aligned}$
$V_{0} \quad$ scale of suction velocity

\section{Introduction}

The study of flow problems which involve the interaction of several phenomena has a wide range of applications in the field of science and technology. One of such studies is related to the effect of free convection MHD flow, which plays an important role in Agriculture, Engineering and Petroleum industries. The problem of free convection under the influence of magnetic field has attracted many researchers in view of its application in Geophysics, Astrophysics, geological formations, and thermal recovery of oil, and in assessment of aquifers, geothermal reservoirs and underground nuclear waste storage site etc. The heat transfer in porous media has great practical importance in geophysics and energy related engineering problems. These include the utilization of geothermal energy, the control of pollutants in ground water, solar power collectors, high performance insulations of buildings, food processing, casting and welding of a manufacturing process etc.

Further, free convection is of interest in the early stages of melting adjacent to a heated surface. There is several industrial and atmospheric applications where the transport processes occurring in nature due to temperature and chemical differences. Heat transfer in porous media is seen both in natural phenomena and in engineered processes. It is replete with the features that are influences of the thermal properties and volume fractions of the materials involved. These features are seen of course as responses to the causes that force the process in to action. For instance many biological materials, whose outermost skin is porous and pervious, saturated or semi saturated with fluids give out and take in heat from their surroundings. The process of heat and mass transfer is encountered in aeronautics, fluid fuel nuclear reactor, chemical process industries and many engineering applications in which the fluid is the working medium. The applications are often found in situation the such as fiber and granules insulation, geothermal systems in the heating and cooling chamber, fossil fuel combustion, energy processes and Astro-physical flows. Further, the magneto-convection place an important role on the control of mountain iron flow in the steady industrial liquid metal cooling in nuclear reactors and magnetic separation of molecular semi conducting materials. A classical example using the nuclear power stations is separation of Uranium U235 from U238 by gases diffusion. When mass transfer takes the place in a fluid rest, the mass is transformed purely by molecular diffusion a result identified from concentration gradient. The wide range of its technological and industrial applications has stimulated considerable amount of interest in the study of heat and mass transfer in convection flows.

Free convection flow is often encountered in cooling of nuclear reactors or in the study of structure of stars and planets. Along with the free convection flow the phenomenon of mass transfer is also very common in the theories of stellar structure. The study of convective flow with mass transfer along a vertical porous plate is receiving considerable attention of many researchers because of its varied applications in the field of cosmical and geophysical sciences. Permeable porous plates are used in the filtration processes and also for a heated body to keep its temperature constant and to make the heat insulation of the surface more effective. The study of stellar structure on the solar surface is connected with mass transfer phenomena. Its origin is attributed to difference in temperature caused by the non-homogeneous production of heat, which in many cases can rest not only in the formation of convective currents but also in violent explosions. Mass transfer certainly occurs within the mantle and cores of planets of the size of or larger than the earth. It is therefore interesting to investigate this phenomenon and to study in particular, the case of mass transfer on the free convection flow.

The study of flow and heat transfer for an electrically conducting polar fluid past a porous plate under the influence of a magnetic field has attracted the interest of many investigators in view of its applications in many engineering problems such as magneto-hydrodynamic (MHD) generator, plasma studies, nuclear reactors, oil exploration, geothermal energy extractions and the boundary layer control in the field of aerodynamics (Soundalgekar et. al., 1977). Also, free convection flows are of great interest in a number of industrial applications such as fiber and granular insulation, geothermal etc. Magneto-hydrodynamic has attracted the attention of a large number of scholars due to its diverse applications. In astrophysics and geophysics, it is applied to study the stellar and solar structures, interstellar matter, radio propagation through the ionosphere etc. In engineering, it finds its application in MHD pumps, MHD bearings etc. Convection in porous media has applications in geothermal energy recovery, oil extraction, thermal energy storage and flow through filtering

Finite element analysis for unsteady MHD heat and mass transfer free convection flow of polar fluids past a vertical... 
devices (Nield and Bejan, 1998). From technological point of view, MHD free convection flows have significant applications in the field of stellar and planetary magneto-sphere, aeronautics, chemical engineering and electronics on account of their varied importance, these flows have been studied by several authors notable amongst them are Shercliff (1965), Ferraro and Plumpton (1966) and Cramer (1973) Also, many transport processes exist in industries and technology where the transfer of heat and mass occurs simultaneously as a result of thermal diffusion and diffusion of chemical species. In addition, polar fluids are fluids with microstructure belonging to a class of fluids with non-symmetrical stress tensor. Physically, they represent fluids consisting of randomly oriented particles suspended in a viscous medium (Aero et. al., 1965, Dep, 1965, Lukaszewicz, 1999, Eringen, 1966 and 1972, Hassanien et. al., 1999, Kim, 2001). Gribben(1965) considered the MHD boundary layer flow over a semi-infinite plate with an aligned magnetic field in the presence of a pressure gradient. He has obtained solutions for large and small magnetic Prandtl numbers using the method of matched asymptotic expansion. Takhar and Ram (1991) studied the effects of Hall current on hydro-magnetic free convection boundary layer flow via a porous medium past a plate, using harmonic analysis. Takhar and Ram (1994) also studied the MHD free convection heat transfer of water at $4^{0} \mathrm{C}$ through a porous medium. Soundalgekar (1973) obtained approximate solutions for the two-dimensional flow of an incompressible, viscous fluid past an infinite porous vertical plate with constant suction velocity normal to the plate, the difference between the temperature of the plate and the free stream is moderately large causing the free convection currents.

Raptis and Kafousias (1982) studied the influence of a magnetic field upon the steady free convection flow through a porous medium bounded by an infinite vertical plate with a constant suction velocity, and when the plate temperature is also constant. Raptis (1986) studied mathematically the case of time-varying twodimensional natural convective heat transfer of an incompressible, electrically conducting viscous fluid via a highly porous medium bounded by an infinite vertical porous plate. Malga et. al. (2011) has studied viscous dissipation effects on unsteady free convection and mass transfer flow past an accelerated vertical porous plate with suction.

Singh (2001) analyzed the MHD free convection and mass transfer flow with heat source and thermal diffusion. The paper deals with the study of free convection and mass transfer flow of an incompressible, viscous and electrically conducting fluid past a continuously moving infinite vertical plate in the presence of large suction and under the influence of uniform magnetic field considering heat source and thermal diffusion. Kim (2000) considered the unsteady magneto-hydrodynamic convective heat transfer past a semi-infinite vertical porous moving plate with variable suction. Khandelwal et. al. (2003) studied the effect of couple stresses on the flow through a porous medium with variable permeability in slip flow regime.

However, most of the previous works assume that the plate is at rest. Saxena and Dubey (2011) considered the case of a semi-infinite moving porous plate with a constant velocity in the longitudinal direction when the magnetic field is imposed transversely to the plate. We also consider the free stream to consist of a mean velocity and temperature with a superimposed exponentially variation with time. In view of the applications of free convective phenomenon, heat source and thermal diffusion, in the present work it is proposed to study the unsteady two dimensional MHD free convective heats and mass transfer of polar fluids past a semi-infinite vertical moving porous plate via a porous medium taking into the account combined effect of heat source and thermal diffusion. Recently Malga.et.al (2013) studied Effects of hall current on an unsteady MHD flow of heat and mass transfer along a porous flat plate with chemical reaction and viscous dissipation.

In general, the study of Darcian porous MHD is very complicated. It is necessary to consider in detail the distribution of velocity and temperature distributions across the boundary layer. The present study is analyzed by using the Galerkin finite element method for the unsteady two-dimensional magneto-hydrodynamics heat and transfer free convection flow of an incompressible, viscous, electrically conducting fluid past a semi-infinite vertical porous plate embedded in a porous medium with thermal diffusion and heat generation is considered. Representative results for the velocity, angular velocity, temperature and concentration profiles have been displayed graphically showing the effect of several governing parameters entering into the problem.

\section{Mathematical Formulation}

We consider the two-dimensional unsteady free convective heat and mass transfer flow of a laminar, incompressible fluid past a semi-infinite vertical porous moving plate embedded in a porous medium and subjected to a transverse magnetic field in the presence of a pressure gradient. It is assumed that there is no 
applied voltage which implies the absence of an electric field. The transversely applied magnetic field and magnetic Reynolds number are very small and hence the induced magnetic field is negligible (Cowling, 1957). Viscous and Darcy's resistance terms are taken into account with constant permeability of the porous medium. The MHD term is derived from an order of magnitude analysis of the full Navier-Stokes equations. It is assumed here that the hole size of the porous plate is significantly larger than a characteristic microscopic length scale of the porous medium. We regard the porous medium as an assemblage of small identical spherical particles fixed in space, the flow variables are functions of $y^{*}$ and $t^{*}$ only.

Under these conditions, the governing equations, i.e., the mass, momentum, energy conservation and diffusion equations can be written in a Cartesian frame of reference as:

Continuity: $\quad \frac{\partial \mathrm{V}^{*}}{\partial \mathrm{y}^{*}}=0$

Linear momentum:

$\frac{\partial u^{*}}{\partial t^{*}}+V^{*} \frac{\partial u^{*}}{\partial y^{*}}=-\frac{1}{\rho} \frac{\partial p^{*}}{\partial x^{*}}+\left(v+v_{r}\right) \frac{\partial^{2} u^{*}}{\partial y^{*}}+\mathrm{g} \beta_{f}\left(T-T_{\infty}\right)+\mathrm{g} \beta_{c}\left(C-C_{\infty}\right)-v \frac{u^{*}}{K^{*}}-\frac{\sigma}{\rho} B_{0}^{2} u^{*}+2 v_{r} \frac{\partial \omega^{*}}{\partial y^{*}}$

Angular momentum: $\rho j^{*}\left(\frac{\partial \omega^{*}}{\partial t^{*}}+V^{*} \frac{\partial \omega^{*}}{\partial y^{*}}\right)=\gamma \frac{\partial^{2} \omega^{*}}{\partial y^{* 2}}$

Energy:

$$
\frac{\partial T}{\partial t^{*}}+V^{*} \frac{\partial T}{\partial y^{*}}=\frac{k}{\rho \mathrm{C}_{\mathrm{p}}} \frac{\partial^{2} T}{\partial y^{*}}+\frac{\mathrm{S}^{*}}{\rho \mathrm{C}_{\mathrm{p}}}\left(T-T_{\infty}\right)
$$

Diffusion:

$$
\frac{\partial C}{\partial t^{*}}+V^{*} \frac{\partial C}{\partial y^{*}}=D \frac{\partial^{2} C}{\partial y^{*}}+\mathrm{D}_{1} \frac{\partial^{2} T}{\partial y^{* 2}}
$$

where $x^{*}$ and $y^{*}$ are the dimensional distances longitudinal and perpendicular to the plate respectively $u^{*}$ and $v^{*}$ the components of dimensional velocities along the $x^{*}$ and $y^{*}$ directions respectively, $K^{*}$ the permeability of the porous medium, $\sigma$ the electrical conductivity of the fluid, $B_{0}$ the magnetic induction, $j^{*}$ the micro-inertia density, $\omega^{*}$ the component of the angular velocity vector normal to the $x y$-plane, $S^{*}$ is the coefficient of heat source, $D$ is the chemical molecular diffusivity and $\mathrm{D}_{1}$ is the thermal diffusivity.

The third term on the RHS of the momentum equation (2) denotes buoyancy effects, the fourth is the bulk matrix linear resistance, i.e. Darcy term and the fifth is the MHD term. Also, Darcy dissipation term is neglected because it is of the same order-of-magnitude as the viscous dissipation term. It is assumed that the porous plate moves with constant velocity $\left(u_{p}^{*}\right)$ in the longitudinal direction and the free stream velocity $\left(U_{\infty}^{*}\right)$ follows an exponentially increasing or decreasing. We also assume that the plate temperature $(T)$ and suction velocity $\left(v^{*}\right)$ vary exponentially with time.

Under the above assumptions the appropriate boundary conditions for the velocity, temperature and concentration fields are at

$u^{*}=u_{p}^{*}, T=T_{w}+\varepsilon\left(T_{w}-T_{\infty}\right) e^{n^{*} t^{*}}, C=C_{w}+\varepsilon\left(C_{w}-C_{\infty}\right) e^{n^{*} t^{*}}, \frac{\partial \omega^{*}}{\partial y^{*}}=-\frac{\partial^{2} u^{*}}{\partial y^{* 2}}$ at $\mathrm{y}^{*}=0$

$u^{*} \rightarrow U_{\infty}^{*}=U_{0}\left(1+\varepsilon e^{n^{*} t^{*}}\right), T \rightarrow T_{\infty}, C \rightarrow C_{\infty}, \omega^{*} \rightarrow 0$ asy $y^{*} \rightarrow \infty$

in which $n^{*}$ is a scalar constant and $U_{0}$ is a scale of free stream velocity.

From the continuity equation (1) it is clear that the suction velocity normal to the plate is a function of time only and we shall take it in the form:

$V^{*}=-V_{0}\left(1+\varepsilon A e^{n^{*} t^{*}}\right)$

where $A$ is a real positive constant $\varepsilon$ and $\varepsilon A$ small less than unity and $V_{0}$ is a scale of suction velocity which has non-zero positive constant. Outside the boundary layer equation (2) gives $-\frac{1}{\rho} \frac{\mathrm{d} p^{*}}{\mathrm{~d} x^{*}}=\frac{d U_{\infty}^{*}}{\mathrm{~d} t^{*}}+\frac{v}{\mathrm{~K}^{*}} \mathrm{U}_{\infty}^{*}+\frac{\sigma}{\rho} B_{0}^{2} U_{\infty}^{*}$

We now introduce the dimensionless variables as follows: 


$$
\begin{gathered}
u=\frac{u^{*}}{U_{0}}, V=\frac{v^{*}}{V_{0}}, y=\frac{y^{*} V_{0}}{v}, U_{\infty}=\frac{U_{\infty}{ }^{*}}{U_{0}}, U_{p}=\frac{u_{p}^{*}}{U_{0}}, \omega=\frac{v}{U_{0} V_{0}} \omega^{*}, t=\frac{t^{*} V_{0}^{2}}{v}, \\
\theta=\frac{\left(T-T_{\infty}\right)}{\left(T_{w}-T_{\infty}\right)}, \quad \emptyset=\frac{\left(C-C_{\infty}\right)}{\left(C_{w}-C_{\infty}\right)}, n=\frac{n^{*} v}{V_{0}^{2}}, K=\frac{K^{*} V_{0}^{2}}{v^{2}}, j=\frac{V_{0}^{2}}{v^{2}} j^{*},
\end{gathered}
$$

Sc $=\frac{v}{\mathrm{D}}$, is the Schmidth number, $P_{r}=\frac{v \rho C_{p}}{\mathrm{k}}=\frac{\mu C_{p}}{\mathrm{k}}$ is the Prandtl number,

$$
\begin{aligned}
& G_{r}=\frac{v g \beta_{\mathrm{f}}\left(\mathrm{T}_{\mathrm{w}}-\mathrm{T}_{\infty}\right)}{\mathrm{U}_{0} \mathrm{~V}_{0}^{2}} \text {, is the Grashof number for heat transfer, } \\
= & \frac{v g \beta_{\mathrm{f}}\left(\mathrm{C}_{\mathrm{w}}-\mathrm{C}_{\infty}\right)}{\mathrm{U}_{0} \mathrm{~V}_{0}^{2}} \text {, is the Modified Grashof number for mass transfer, }
\end{aligned}
$$

$M=\frac{\sigma B_{0}^{2} v}{\rho \mathrm{V}_{0}^{2}}$ is the Magnetic field parameter, $S=\frac{S^{*} v}{\rho C_{p} \mathrm{~V}_{0}^{2}}$ is the heat source parameter,

$$
\mathrm{A}_{\mathrm{T}}=\frac{\mathrm{D}_{1}}{v} \frac{\left(T_{w}-T_{\infty}\right)}{\left(C_{w}-C_{\infty}\right)} \text { is the thermal diffusion parameter, }
$$

Furthermore, the spin-gradient viscosity $\gamma$ which gives some relationship between the coefficients of viscosity and micro-inertia is defined as

$\gamma=\left(\mu+\frac{\Lambda}{2}\right) j^{*}=\mu j^{*}\left(1+\frac{1}{2} \beta\right)$

where $\beta$ denotes the dimensionless viscosity ratio defined as follows:

$\beta=\frac{\Lambda}{\mu}$

in which $\Lambda$ is the coefficient of gyro-viscosity (or vortex viscosity).

In view of Equations (8)-(12), the governing equations (2)-(5) reduce to the following non-dimensional form:

$\frac{\partial u}{\partial t}-\left(1+\varepsilon A e^{n t}\right) \frac{\partial u}{\partial y}=\frac{d U_{\infty}}{d t}+(1+\beta) \frac{\partial^{2} u}{\partial y^{2}}+G_{r} \theta+G_{c} \emptyset+N\left(U_{\infty}-u\right)+2 \beta \frac{\partial \omega}{\partial y}$

$\frac{\partial \omega}{\partial t}-\left(1+\varepsilon A e^{n t}\right) \frac{\partial \omega}{\partial y}=\frac{1}{\eta} \frac{\partial^{2} \omega}{\partial y^{2}}$

$\frac{\partial \theta}{\partial t}-\left(1+\varepsilon A e^{n t}\right) \frac{\partial \theta}{\partial y}=\frac{1}{\operatorname{Pr}} \frac{\partial^{2} \theta}{\partial y^{2}}+\mathrm{S} \theta$

$\frac{\partial \emptyset}{\partial t}-\left(1+\varepsilon A e^{n t}\right) \frac{\partial \emptyset}{\partial y}=\frac{1}{S c} \frac{\partial^{2} \emptyset}{\partial y^{2}}+\mathrm{A}_{\mathrm{T}} \frac{\partial^{2} \theta}{\partial y^{2}}$

where $N=\left(M+\frac{1}{K}\right), \eta=\frac{\mu j^{*}}{\gamma}=\frac{2}{2+\beta}$

The boundary conditions (6) and (7) are then given by the following dimensionless form: $\left.\begin{array}{c}u \rightarrow U_{p}, \theta=1+\varepsilon e^{n t}, \emptyset=1+\varepsilon e^{n t}, \frac{\partial \omega}{\partial y}=-\frac{\partial^{2} u}{\partial y^{2}} \text { aty }=0 \\ u \rightarrow U_{\infty}, \theta \rightarrow 0, \emptyset \rightarrow 0, \omega \rightarrow 0 \text { asy } \rightarrow \infty\end{array}\right\}$

where $U_{p}$ is the plate moving velocity.

\section{Numerical Method of Solution}

In order to reduce the above system of partial differential equations to a system of dimensionless form we may represent the linear and angular velocities, temperature and concentration by applying the Galerkin finite element method for Equation (13) over a typical two-noded linear element $(e)\left(y_{j} \leq y \leq y_{k}\right)$ is

$$
\begin{gathered}
u=N . \emptyset, N=\left[N_{j}, N_{k}\right], \emptyset=\left[\begin{array}{l}
u_{j} \\
u_{k}
\end{array}\right], N_{j}=\frac{y_{k}-y}{l}, \quad N_{k}=\frac{y-y_{j}}{l}, l=y_{k}-y_{j}=h, \\
\int_{y_{j}}^{y_{k}} N^{T}\left[(1+\beta) \frac{\partial^{2} u}{\partial y^{2}}+\left(1+\varepsilon A e^{n t}\right) \frac{\partial u}{\partial y}-\frac{\partial u}{\partial t}+\frac{d U_{\infty}}{d t}+N\left(U_{\infty}-u\right)+\left(G_{r} \theta+G_{c} \emptyset\right)+2 \beta \frac{\partial \omega}{\partial y}\right] d y \\
\int_{y_{j}}^{y_{k}}\left[(1+\beta) \frac{\partial N}{\partial y} \cdot \frac{\partial u}{\partial y}-N^{T}\left(\left(1+\varepsilon A e^{n t}\right) \frac{\partial u}{\partial y}-\frac{\partial u}{\partial t}+\frac{d U_{\infty}}{d t}+N\left(U_{\infty}-u\right)+\left(G_{r} \theta+G_{c} \emptyset\right)+2 \beta \frac{\partial \omega}{\partial y}\right)\right] d y=0 \\
\int_{y_{j}}^{y_{k}}\left[(1+\beta) \frac{\partial N}{\partial y} \cdot \frac{\partial u}{\partial y}-N^{T}\left(\left(1+\varepsilon A e^{n t}\right) \frac{\partial u}{\partial y}-\frac{\partial u}{\partial t}-N u+R\right)\right] d y=0
\end{gathered}
$$


where $R=\left((\mathrm{N}+\mathrm{n}) \varepsilon e^{n t}+\mathrm{N}+G_{r} \theta+G_{c} \emptyset+2 \beta \frac{\partial \omega}{\partial y}\right)$

The element equation given by

$$
\begin{gathered}
\int_{y_{j}}^{y_{k}}(1+\beta)\left[\begin{array}{l}
N_{j}^{\prime} N_{j}^{\prime} N_{j}^{\prime} N_{k}^{\prime} \\
N_{k}^{\prime} N_{j}^{\prime} N_{k}^{\prime} N_{k}^{\prime}
\end{array}\right]\left[\begin{array}{l}
u_{j} \\
u_{k}
\end{array}\right] d y-\left(1+\varepsilon A e^{n t}\right)\left[\begin{array}{l}
N_{j} N_{j}^{\prime} N_{j} N_{k}^{\prime} \\
N_{k} N_{j}^{\prime} N_{k} N_{k}^{\prime}
\end{array}\right]\left[\begin{array}{l}
u_{j} \\
u_{k}
\end{array}\right] d y+\left[\begin{array}{cc}
N_{j} N_{j} & N_{j} N_{k} \\
N_{k} N_{j} & N_{k} N_{k}
\end{array}\right]\left[\begin{array}{l}
\dot{u}_{j} \\
\dot{u_{k}}
\end{array}\right] d y \\
+\mathrm{N}\left[\begin{array}{ll}
N_{j} N_{j} & N_{j} N_{k} \\
N_{k} N_{j} & N_{k} N_{k}
\end{array}\right]\left[\begin{array}{l}
u_{j} \\
u_{k}
\end{array}\right] d y-R\left[\begin{array}{l}
N_{j} \\
N_{k}
\end{array}\right] d y=0 \\
\int_{y_{j}}^{y_{k}}\left(S+\mathrm{A}_{\mathrm{e}}\right) d y=\int_{y_{j}}^{y_{k}} R^{*} d y
\end{gathered}
$$

where

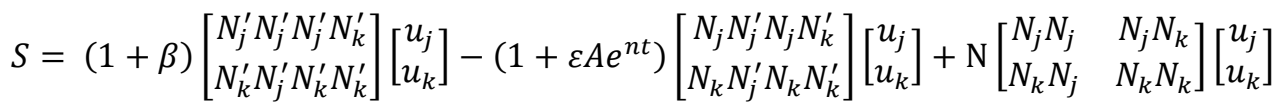

$$
\begin{aligned}
& \mathrm{A}_{\mathrm{e}}=\left[\begin{array}{cc}
N_{j} N_{j} & N_{j} N_{k} \\
N_{k} N_{j} & N_{k} N_{k}
\end{array}\right]\left[\begin{array}{c}
\dot{u}_{j} \\
\dot{u_{k}}
\end{array}\right] \text { and } R^{*}=R\left[\begin{array}{l}
N_{j} \\
N_{k}
\end{array}\right]
\end{aligned}
$$

Here the prime and dot denote differentiation with respect to $\mathrm{y}$ and $\mathrm{t}$. we obtain

$S=\frac{(1+\beta)}{l}\left[\begin{array}{rr}1 & -1 \\ -1 & 1\end{array}\right]\left[\begin{array}{l}u_{j} \\ u_{k}\end{array}\right]-\frac{\left(1+\varepsilon A e^{n t}\right)}{2}\left[\begin{array}{ll}-1 & 1 \\ -1 & 1\end{array}\right]\left[\begin{array}{l}u_{j} \\ u_{k}\end{array}\right]+N \frac{l}{6}\left[\begin{array}{ll}2 & 1 \\ 1 & 2\end{array}\right]\left[\begin{array}{l}u_{j} \\ u_{k}\end{array}\right]$

$\mathrm{A}_{\mathrm{e}}=\frac{l}{6}\left[\begin{array}{ll}2 & 1 \\ 1 & 2\end{array}\right]\left[\begin{array}{c}\dot{u}_{j} \\ \dot{u}_{k}\end{array}\right] \operatorname{andR}^{*}=R \frac{l}{2}\left[\begin{array}{l}1 \\ 1\end{array}\right]$

We write the element equation for the elements $y_{i-1} \leq y \leq y_{i}$ and $y_{i} \leq y \leq y_{i+1}$. Assembling these element equations, we get

$$
\begin{array}{r}
\frac{(1+\beta)}{l}\left[\begin{array}{rrr}
1 & -1 & 0 \\
-1 & 2 & -1 \\
0 & -1 & 1
\end{array}\right]\left[\begin{array}{c}
u_{i-1} \\
u_{i} \\
u_{i+1}
\end{array}\right]-\frac{\left(1+\varepsilon A e^{n t}\right)}{2}\left[\begin{array}{rrr}
-1 & 1 & 0 \\
-1 & 0 & 1 \\
0 & -1 & 1
\end{array}\right]\left[\begin{array}{c}
u_{i-1} \\
u_{i} \\
u_{i+1}
\end{array}\right]+\mathrm{N} \frac{l}{6}\left[\begin{array}{ccc}
2 & 1 & 0 \\
1 & 4 & 1 \\
0 & 1 & 2
\end{array}\right]\left[\begin{array}{c}
u_{i-1} \\
u_{i} \\
u_{i+1}
\end{array}\right]+ \\
\frac{l}{6}\left[\begin{array}{ccc}
2 & 1 & 0 \\
1 & 4 & 1 \\
0 & 1 & 2
\end{array}\right]\left[\begin{array}{c}
u_{i+1}^{+} \\
u_{i} \\
u_{i+1}^{\cdot}
\end{array}\right]=R \frac{l}{2}\left[\begin{array}{l}
1 \\
2 \\
1
\end{array}\right]
\end{array}
$$

Now put row corresponding to the node i to zero, from Equation (20) the difference schemes with $l=h$ is $\frac{h}{6}\left(\dot{u}_{i-1}+4 \dot{u}_{i}+\dot{u}_{i+1}\right)+\frac{(1+\beta)}{\mathrm{h}}\left(-u_{i-1}+2 u_{i}-u_{i+1}\right)-\frac{\left(1+\varepsilon A e^{n t}\right)}{2}\left(-u_{i-1}+u_{i+1}\right)+\frac{N \mathrm{~h}}{6}\left(u_{i-1}+4 u_{i}+u_{i+1}\right)=R^{*}$

Using the Cranck-Nicolson method to Equation (21), we obtain:

$A_{1} u_{i-1}^{j+1}+A_{2} u_{i}^{j+1}+A_{3} u_{i+1}^{j+1}=A_{4} u_{i-1}^{j}+A_{5} u_{i}^{j}+A_{6} u_{i+1}^{j}+R^{*}$

Similarly, Equations (14), (15), (16) are becoming as follows:

$B_{1} w_{i-1}^{j+1}+B_{2} w_{i}^{j+1}+B_{3} w_{i+1}^{j+1}=B_{4} w_{i-1}^{j}+B_{5} w_{i}^{j}+B_{6} w_{i+1}^{j}$

$C_{1} \theta_{i-1}^{j+1}+C_{2} \theta_{i}^{j+1}+C_{3} \theta_{i+1}^{j+1}=C_{4} \theta_{i-1}^{j}+C_{5} \theta_{i}^{j}+C_{6} \theta_{i+1}^{j}$

$D_{1} \varnothing_{i-1}^{j+1}+D_{2} \varnothing_{i}^{j+1}+D_{3} \varnothing_{i+1}^{j+1}=D_{4} \varnothing_{i-1}^{j}+D_{5} \varnothing_{i}^{j}+D_{6} \varnothing_{i+1}^{j}+R^{* *}$

$A_{1}=(1-6 r(1+\beta)+3 p V+N k), A_{2}=(4+12 r(1+\beta)+4 N k), A_{3}=(1-6 r(1+\beta)-3 p V+N k)$

$A_{4}=(1+6 r(1+\beta)-3 p V-N k), A_{5}=(4-12 r(1+\beta)-4 N k), A_{6}$

$$
=(1+6 r(1+\beta)+3 p V-4 N k)
$$

$B_{1}=\left(1-6 r \frac{1}{\eta}+3 p V\right), B_{2}=\left(4+12 r \frac{1}{\eta}\right), B_{3}=\left(1-6 r \frac{1}{\eta}-3 p V\right)$,

$B_{4}=\left(1+6 r \frac{1}{\eta}-3 p V\right), B_{5}=\left(4-12 r \frac{1}{\eta}\right), B_{6}=\left(1+6 r \frac{1}{\eta}+3 p V\right)$,

$C_{1}=\left(1-6 r \frac{1}{P_{r}}+3 p V-S k\right), C_{2}=\left(4+12 r \frac{1}{P_{r}}-4 S k\right), C_{3}=\left(1-6 r \frac{1}{P_{r}}-3 p V-S k\right)$, 


$$
\begin{gathered}
C_{4}=\left(1+6 r \frac{1}{P_{r}}-3 p V+S k\right), C_{5}=\left(4-12 r \frac{1}{P_{r}}+4 S k\right), C_{6}=\left(1+6 r \frac{1}{P_{r}}+3 p V+4 S k\right), \\
D_{1}=\left(1-6 r \frac{1}{S_{c}}+3 p V\right), D_{2}=\left(4+12 r \frac{1}{S_{c}}\right), D_{3}=\left(1-6 r \frac{1}{S_{c}}-3 p V\right), \\
D_{4}=\left(1+6 r \frac{1}{s_{c}}-3 p V\right), D_{5}=\left(4-12 r \frac{1}{s_{c}}\right), D_{6}=\left(1+6 r \frac{1}{S_{c}}+3 p V\right), \\
R^{* *}=\left(\frac{U_{i+1}-2 U_{i}+U_{i-1}}{h^{2}}\right)
\end{gathered}
$$

Here $r=\frac{k}{h^{2}}$ where $\mathrm{k}, \mathrm{h}$ is mesh sizes along y direction and time direction respectively. Index i refers to space and $\mathrm{j}$ refers to time. The mesh system consists of $\mathrm{h}=0.05$ for velocity profiles and concentration profiles and $\mathrm{k}=0.025$ has been considered for computations. In Equations (23)-(26), taking $\mathrm{i}=1(1) \mathrm{n}$ and using initial and boundary conditions (17), the following system of equation are obtained.

$A_{i} X_{i}=B_{i}, \quad i=1,2,3 \ldots$

Where $A_{i}$ 's are matrices of order $\mathrm{n}$ and $X_{i} a n d B_{i}$ 's are column matrices having n-components. The solution of above system of equations are obtained using Thomas algorithm for velocity, angular velocity, temperature and concentration. Also, numerical solutions for these equations are obtained by MATLAB program. In order to prove the convergence and stability of Galerkin finite element method, the same MAT LAB program was run with slightly changed values of $\mathrm{h}$ and $\mathrm{k}$, no significant change was observed in the values of $u, w, \theta, C$. Hence, the Galerkin finite element method is stable and convergent.

\section{Results and Discussion}

To give good insight of the physical problem addressing in this study, we discussed the effect of various parameters like suction velocity parameter A, viscosity ratio $\beta$, Grashof number Gr, Modified Grashof number Gc, source parameter $S$, permeability parameter $\mathrm{K}$, scalar constant $\varepsilon$, Magnetic field parameter $\mathrm{M}$, thermal diffusion parameter $\mathrm{A}_{\mathrm{T}}$, dimensionless exponential index $\mathrm{n}$, dimensionless time $\mathrm{t}$, plate moving velocity $U_{p}$, Prandtl number $\operatorname{Pr}$, Schmidt number $S c$ on the velocity profiles u, angular velocity profiles $\omega$, temperature profiles $\theta$ and concentration profiles $\varnothing$.

It is observed that the velocity and temperature decrease in water compared with air. The velocity and angular velocity profiles for various values of viscosity ratio $\beta$ for the fluids air $(\operatorname{Pr}=0.71)$ and water $(\operatorname{Pr}=7.0)$ are displayed in Fig. 1. It is observed that the velocity distribution is lower for a Newtonian fluid $(\beta=0)$ with the fixed flow and material parameters, as compared with a polar fluid when the viscosity ratio $\beta$ is less than 0.5 . For a Newtonian fluid $(\beta=0)$, the velocity graph in water is lower than the respective velocity graph in air while this situation is reverse in case of angular velocity. When viscosity ratio $\beta$ takes values larger than 0.5 , however, the velocity distribution shows a decelerating nature near the porous plate. In addition, the angular velocity distributions do not show consistent variations with increment of viscosity ratio $\beta$. The effect of dimensionless exponential index $n$ on the velocity profiles for stationary porous plate is shown in Fig. 2 for the fluids air and water. It is observed that with the increases of exponential index $n$ from 0.1 to 0.4 , the velocity decreases in both the cases.

Fig. 3 displays the velocity and angular velocity profiles across the boundary layer for different values of the plate velocity $U_{p}$. For the fluid air the peak value of velocity across the boundary layer increases near the porous plate as the plate velocity $U_{p}$ increases and the reverse phenomenon is observed from far away the plate. Also, the velocity across the boundary layer increases in water and then decays to the relevant free stream velocity $U_{0}$. However, the angular velocity increases as the plate velocity increases. It is also observed that at some fixed value of $U_{p}$, the angular velocity graph in air is lower than the respective angular velocity graph in water. 


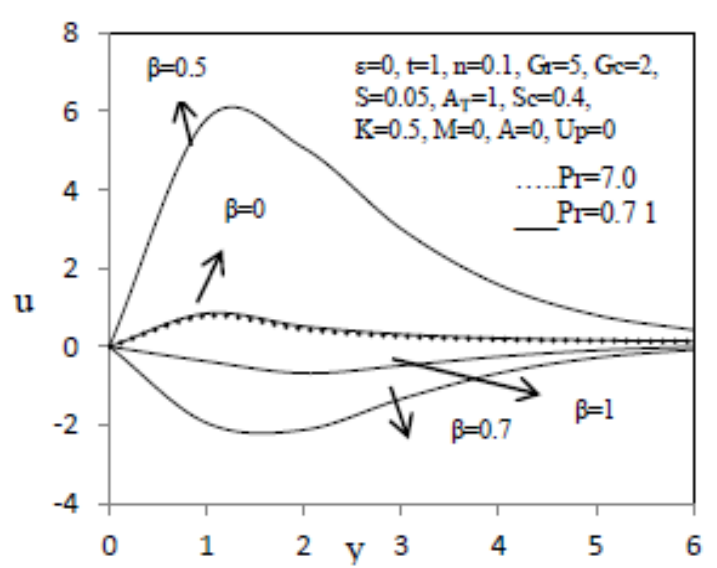

Fig. 1(a): Velocity profiles for different values of viscosity $\boldsymbol{\beta}$

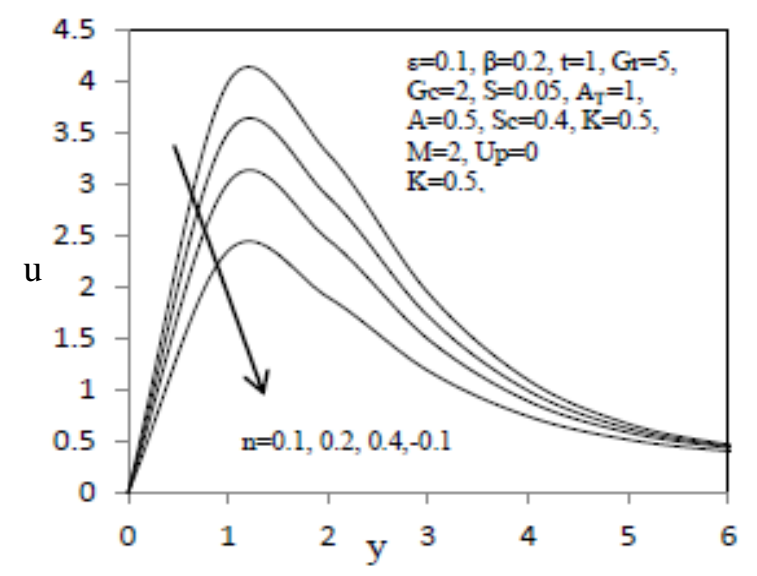

Fig. 2(a): Velocity profiles dimensionless exponential index $n$

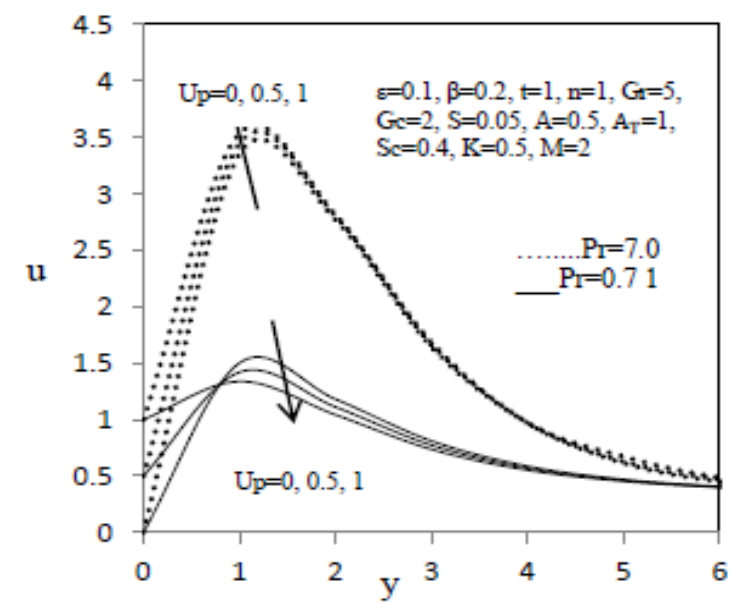

Fig. 3(a): Velocity profiles for different values of Plate moving velocity $\mathbf{U p}$

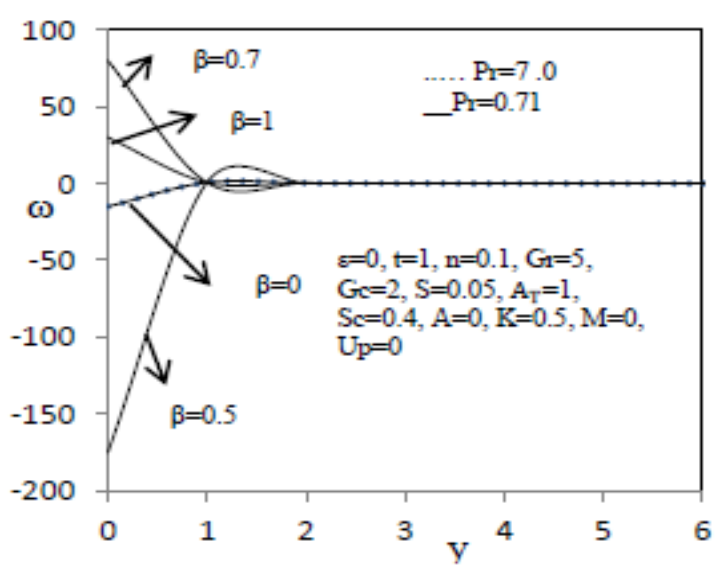

Fig. 1(b): Angular velocity profiles for different values of viscosity $\beta$

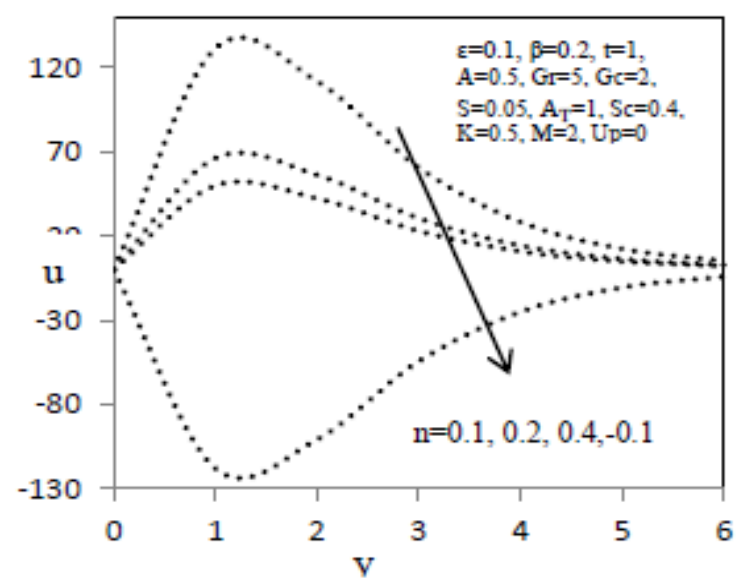

Fig. 2(b): Velocity profiles dimensionless exponential index $\boldsymbol{n}$

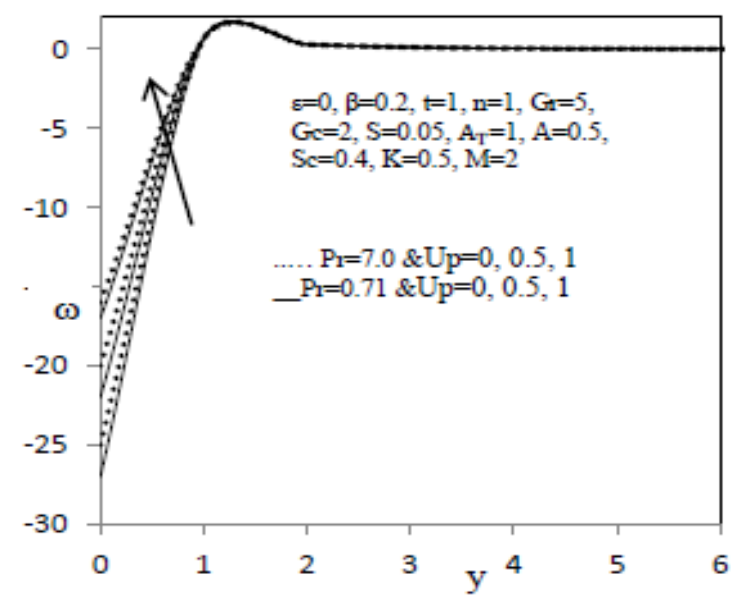

Fig. 3(b): Angular velocity profiles for different values plate moving velocity $\mathbf{U p}$

Fig. 4 depicts the velocity and angular velocity profiles for different values of the magnetic field parameter $M$, in air and water. It is observed that the velocity decreases with the increasing values of magnetic field parameter $M$. This agrees with the expectations, since the magnetic field exerts a retarding force on the free convective flow. Furthermore, it is clear that due to increasing values of $M$, the values of angular velocity on the porous 
plate are decreased in air and increased in water. It is also noticed that for different values of $M$, the angular velocity graphs in air are lower than the respective angular velocity graphs in water.

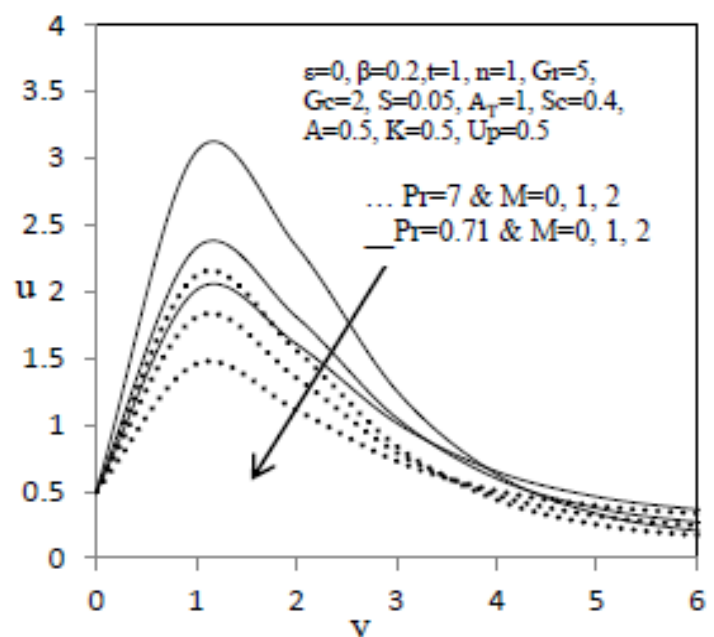

Fig. 4(a): Velocity profiles for different values of magnetic parameter $\boldsymbol{M}$

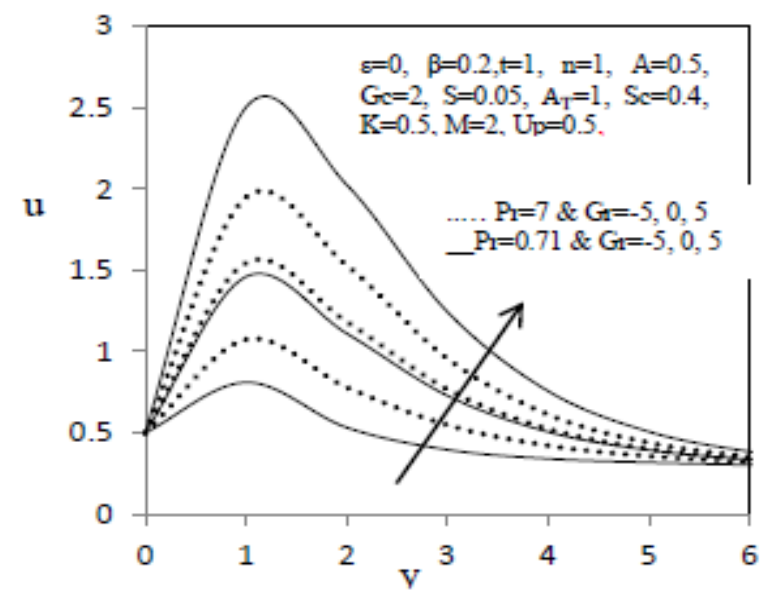

Fig. 5(a): Velocity profiles for different values of Grashof number Gr

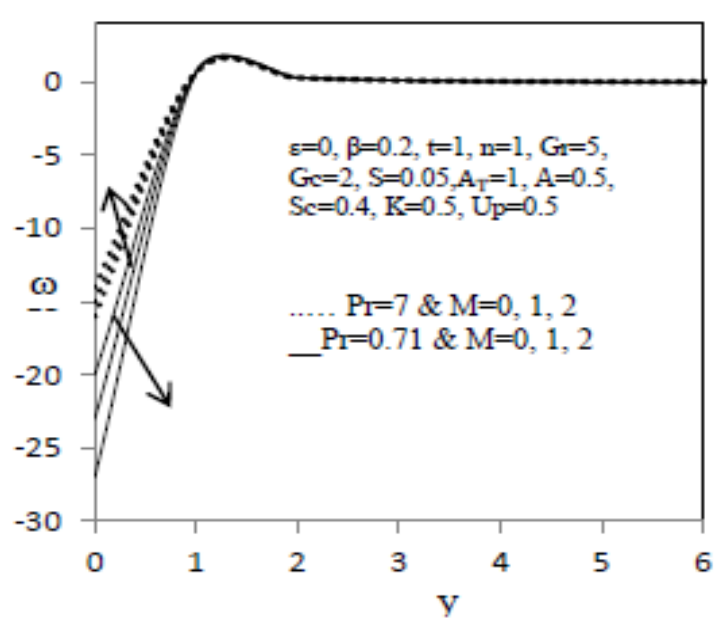

Fig. 4(b): Angular velocity profiles for different of values of magnetic parameter $\boldsymbol{M}$

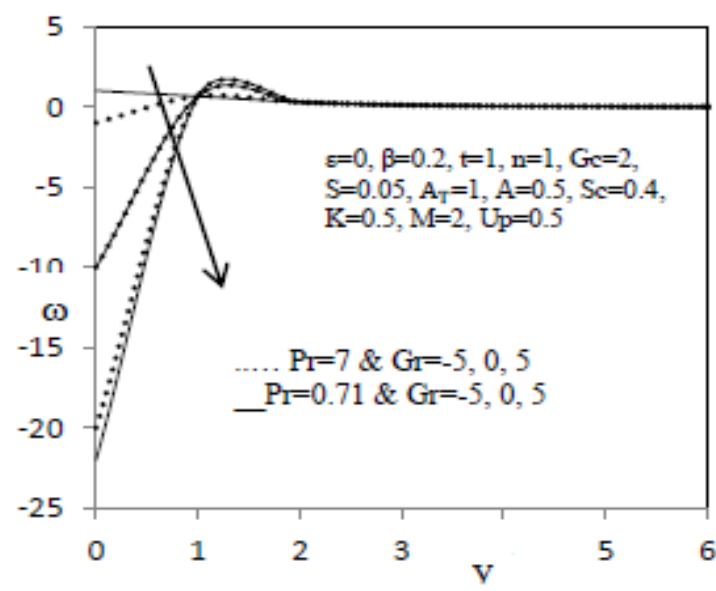

Fig. 5(b): Angular velocity profiles for different values of Grashof number $\mathbf{G r}$

For different values of the Grashof number $G r$, the velocity and angular velocity profiles are depicted in Fig. 5 \& Fig. 6 It is obvious that an increase in $G r$ leads to a rise in the values of velocity, while decreases the angular velocity. Negative values of $G r$, which indicates the heating of the plate, are also taken into account. Also, the large values of $M$ and $G r$ respectively correspond to a strong magnetic field and to a cooling problem that is generally encountered in nuclear engineering in connection with the cooling of reactors. The effect of $G c$ leads to increases the velocity profiles, where it decelerates the angular velocity as noticed from Fig. 6 . It can be seen that the effect of $G r, G c$ is more in air than in water.

The velocity profiles for different values of the permeability parameter $K$ are plotted in Fig. 7. It is clear that the velocity increases due to increasing values of permeability parameter $K$ both in air and water. 


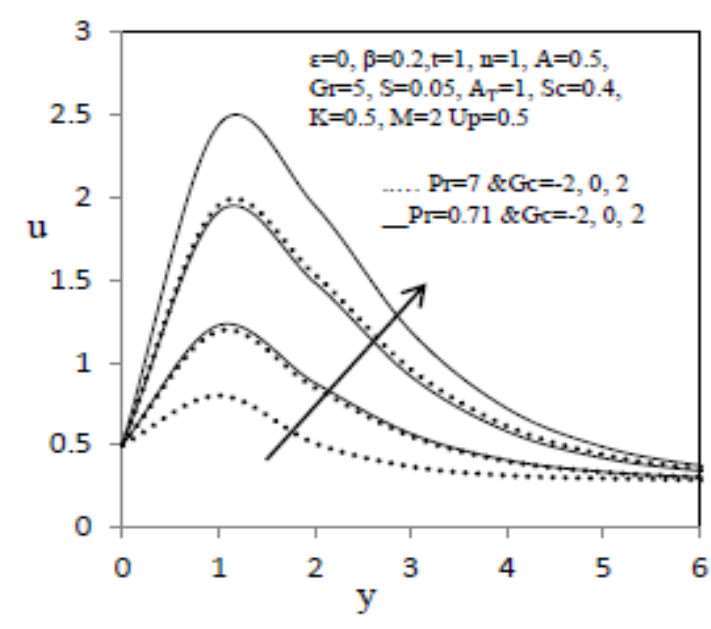

Fig. 6(a): Velocity profiles for different values of modified Grashof number $\mathbf{G c}$

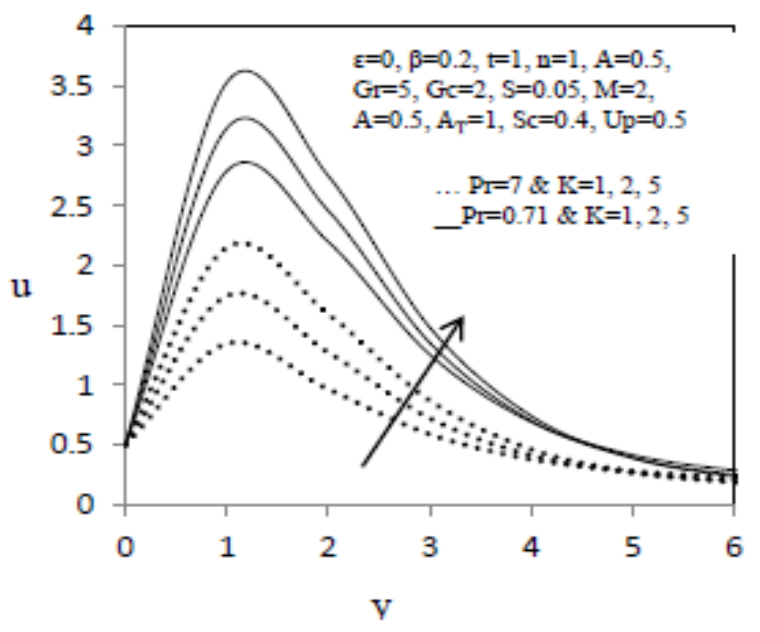

Fig. 7: Velocity profiles for different values of Permeability parameter $\boldsymbol{K}$

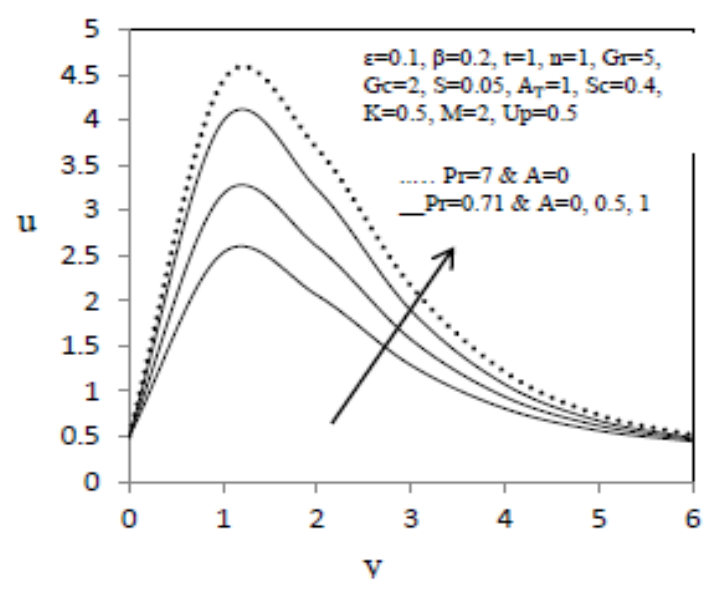

Fig. 9: Velocity profiles for different values of suction velocity parameter $\boldsymbol{A}$

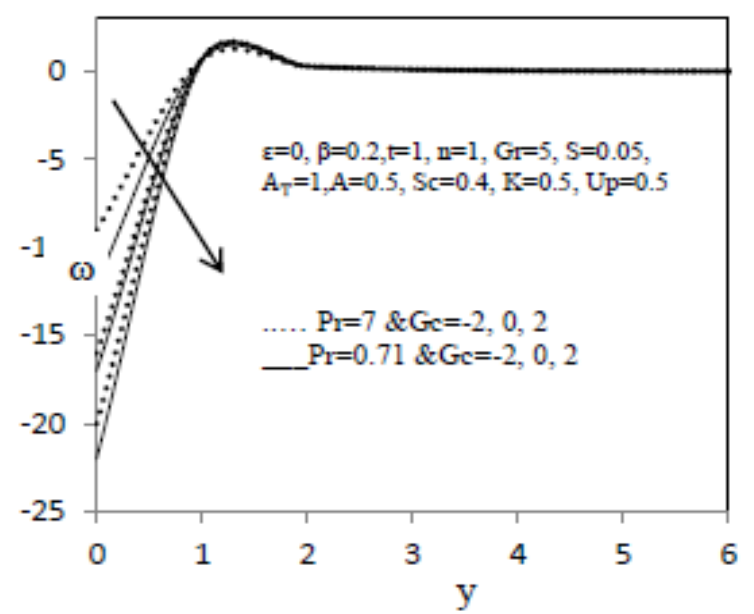

Fig. 6(b): Angular velocity profiles for modified different values of Grashof number $\boldsymbol{G c}$

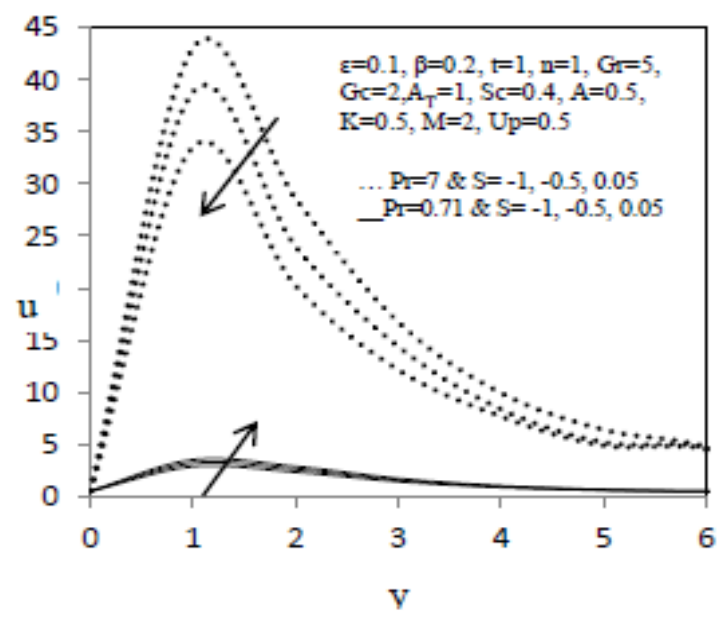

Fig. 8: Velocity profiles for different values of Source parameter $S$

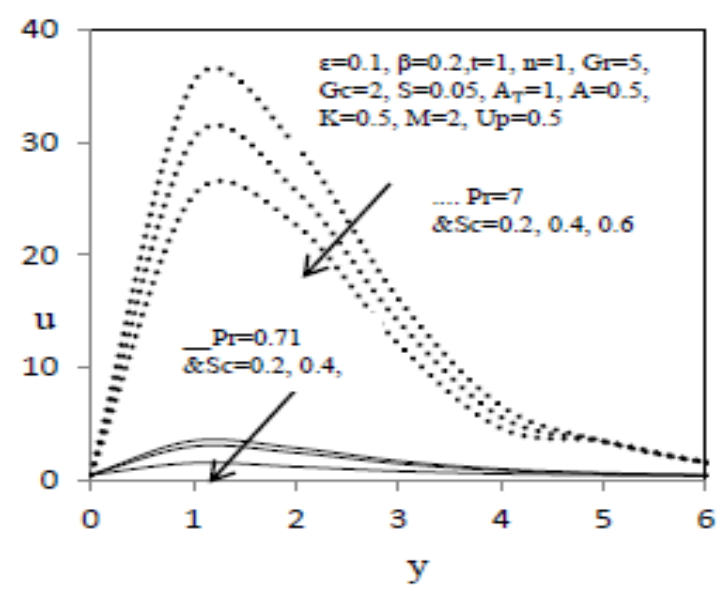

Fig. 10: Velocity profiles for different values of Schmidt number $\boldsymbol{S c}$ 
Fig. 8 demonstrates the velocity profiles for various values of source parameter $S$. Clearly as $S$ increases, the velocity increases in air and decreases in water. It is also observed that the effect of $S$ with water is more than in air on velocity profiles.

The effect of suction velocity parameter $A$ on the velocity is presented in Fig. 9. It is obvious that an increase in $A$ leads to a rise in the values of velocity both in air and water. The velocity profiles for different values of Schmidt number Sc are plotted in Fig. 10, diffusing chemical species of most common interest in air. The numerical results show that the increasing in $S c$ leads to decreasing the velocity distribution across the boundary layer. The results also indicate that the effect of $S c$ on the velocity is higher in water as compared to that in air.

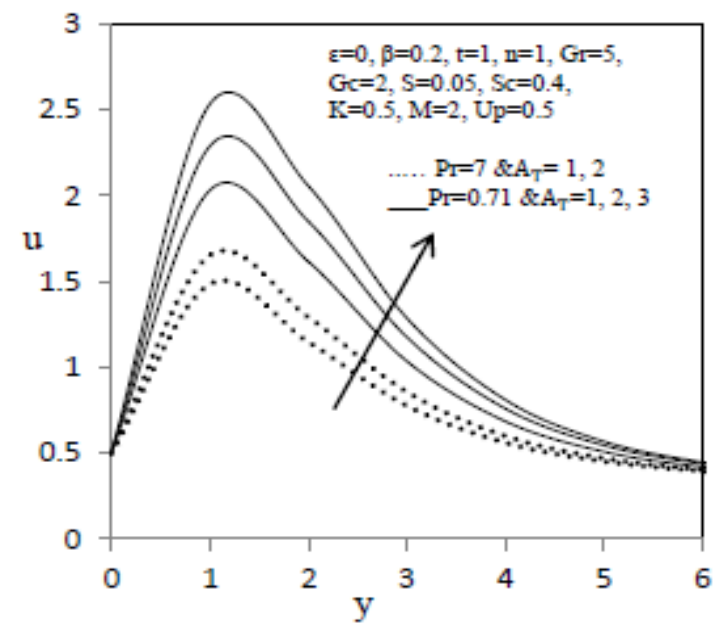

Fig. 11(a): Velocity profiles for different values of thermal diffusion parameter $A_{T}$

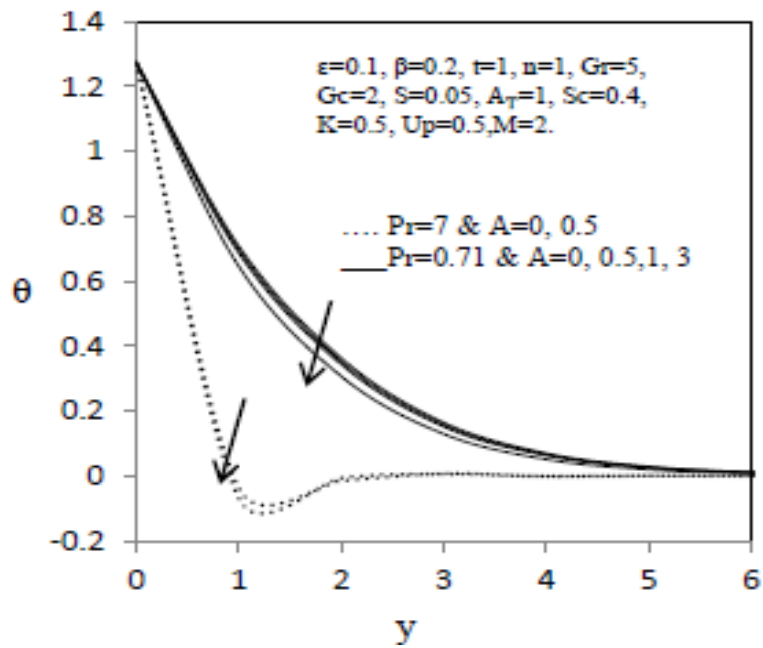

Fig. 12(a): Temperature profiles for different values of suction velocity parameter $\boldsymbol{A}$

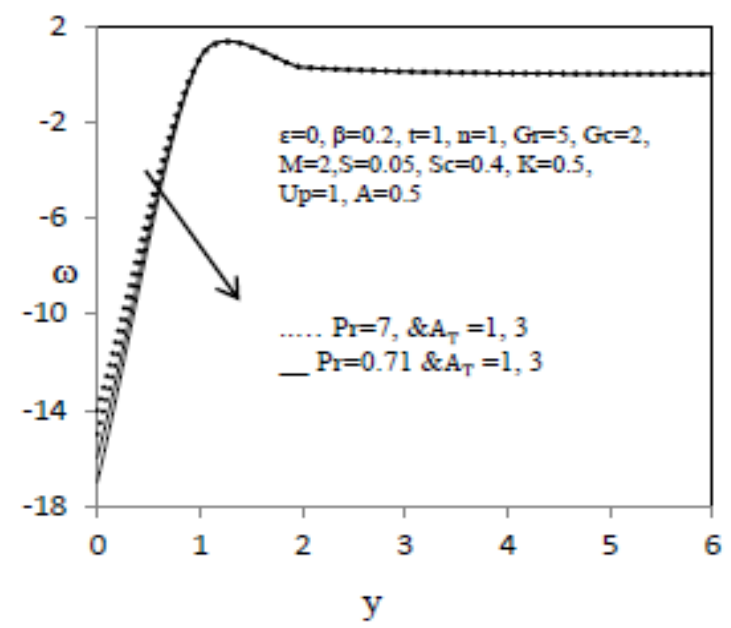

Fig. 11(b):Angular velocity profiles for different values of thermal diffusion parameter $A_{T}$

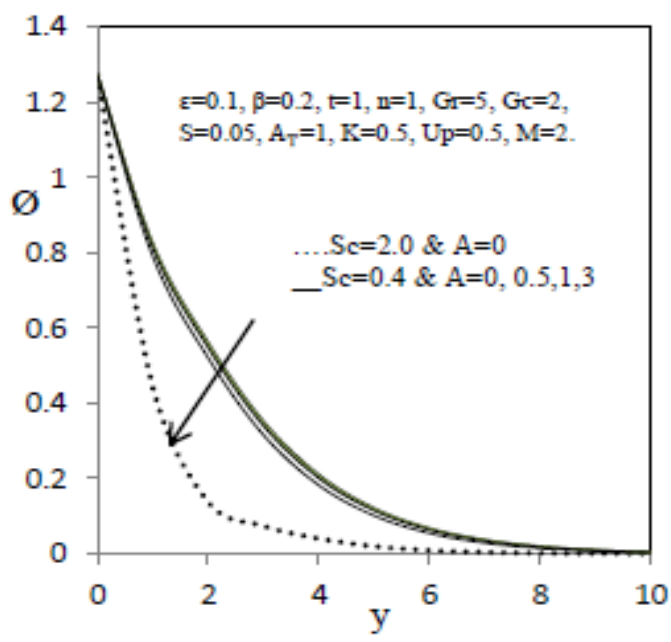

Fig. 12(b): Concentration profiles for different values of $S \mathrm{c}$ and suction velocity parameter $\boldsymbol{A}$

For various values of diffusion parameter $A_{T}$, the velocity and angular velocity profiles are plotted in Fig. 11. As $A_{T}$ increases, the velocity increases both in air and water. Furthermore, it can be seen that with increases in $A_{T}$ the angular velocity decreases for both the fluids. It is clearly seen that the effect of $\mathrm{A}_{\mathrm{T}}$ on angular velocity is less.The variations of the temperature and concentration profiles for different values of $A, t, S, S c, \mathrm{~A}_{\mathrm{T}}$ with given flow and material parameters are shown graphically in Fig. 12-16. It has been observed that the temperature and concentration profiles are lower in water as compared to that in air. It is observed that increasing the $P r$ values a great decrease of the thermal bounder layer. This is good agreement with physical fact that an increasing $\mathrm{Pr}$ 
values leads to decrease the thermal bounder layer thickness. The reason underlying such behaviour is that the higher $P r$ value fluid has lower thermal conductivity. This gives a result in the decrement of thermal boundary layer thickness. The reason is that smaller values of $\mathrm{Pr}$ and $\mathrm{Sc}$ are equivalent to increasing thermal conductivities, and therefore heat is able to diffuse away from the heated surface more rapidly than for higher values of $P r$ and Sc.

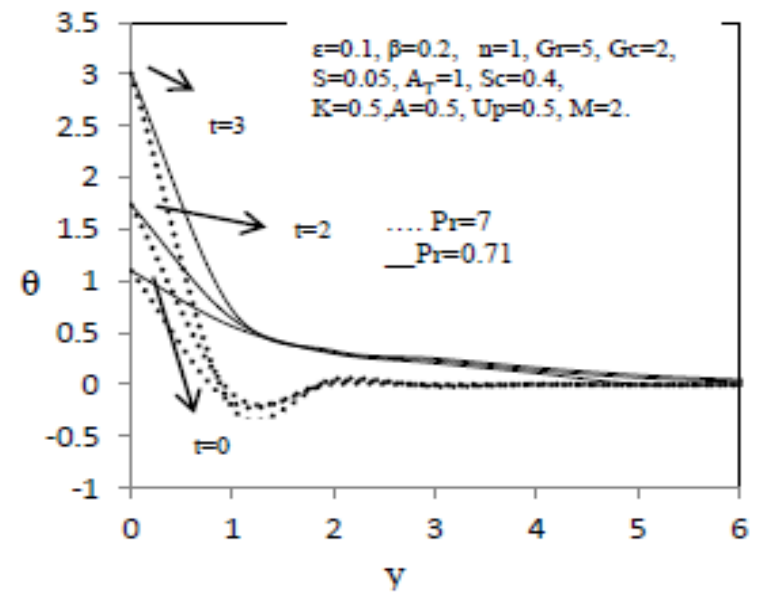

Fig. 13(a): Temperature profiles for different values of dimensionless time $t$

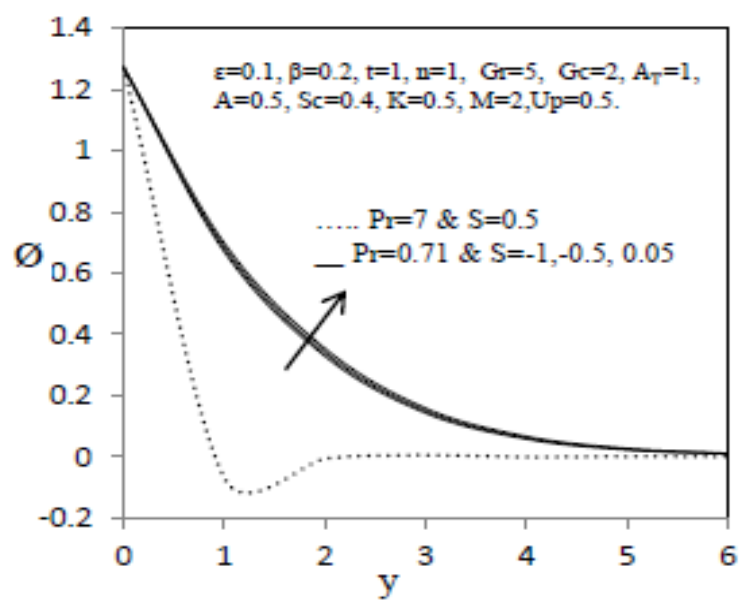

Fig. 14: Concentration profiles for different values of source parameter $S$

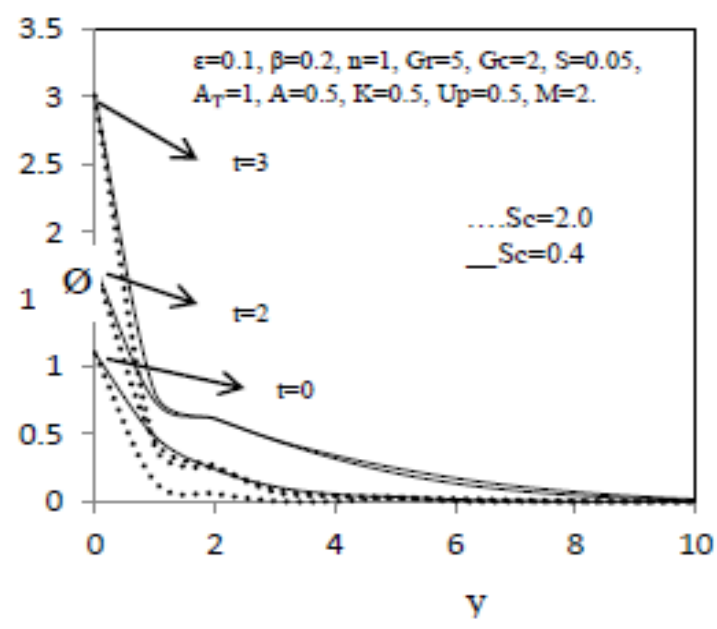

Fig. 13(b): Concentration profiles for different values of dimensionless time $\boldsymbol{t}$

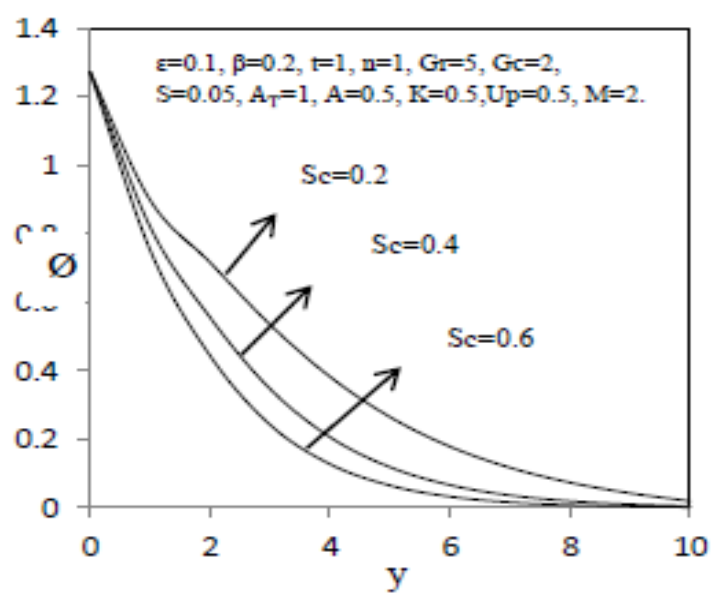

Fig. 15: Concentration profiles for different values of Schmidt number $\mathbf{S c}$

From Fig. 12 it has been observed that the effect of increasing values of suction velocity parameter $A$ results in a decreasing thermal boundary layer thickness approaching to zero. Fig. 13 represents the temperature and concentration profiles for various values of dimensionless time $t$. The temperature and concentration profiles are increase with the time t. Fig. 14 depicts the effect of source parameter Son the temperature. It is noticed that the temperature increases as $S$ increases. The effect of Schmidt number $S c$ on the concentration profiles is shown Fig. 15. The $S c$ effect is leads to increases the concentration profiles are observed form the figure. The concentration profile for different values of diffusion parameter $A_{T}$ is shown in Fig. 16. It is observed that concentration profiles increases with the increase of $\mathrm{A}_{\mathrm{T}}$ the concentration profiles are higher when $S c$ is 0.4 , compared with $S c$ is 2 . The general distributions of velocity and temperature profiles of Newtonian fluid across the boundary layer for a stationary porous plate in the case of absence of magnetic field are shown in Fig. 17. 


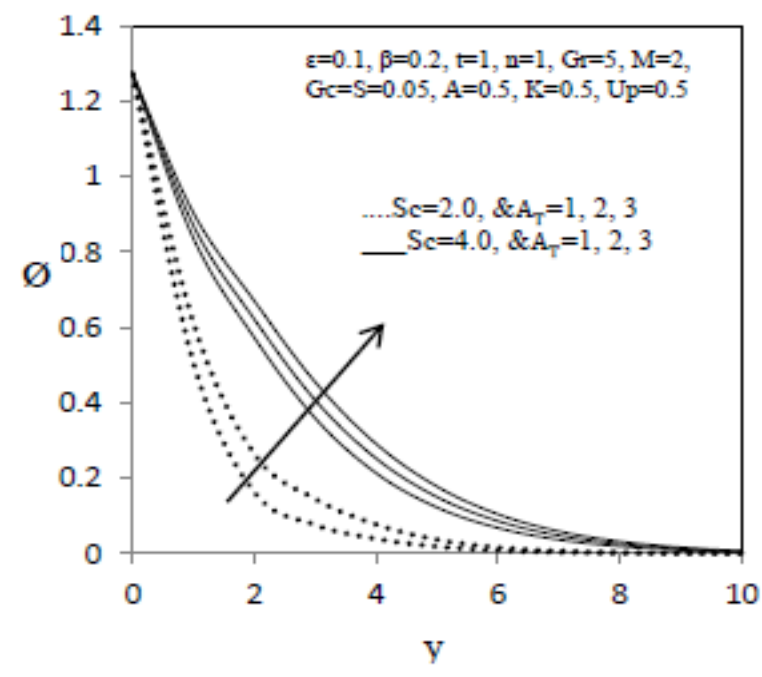

Fig. 16: Concentration profiles for different values of thermal diffusion parameter $\mathrm{A}_{\mathrm{T}}$

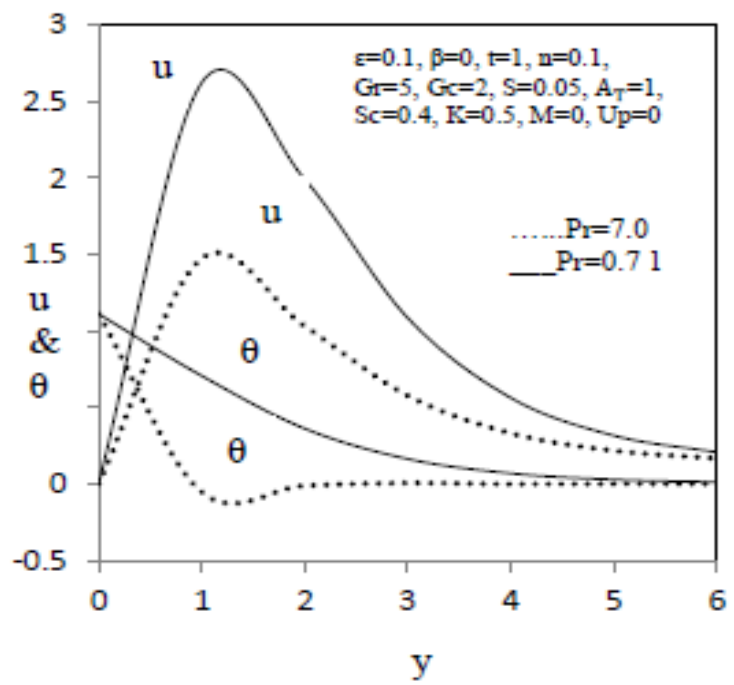

Fig. 17: Distribution of velocity and temperature profiles of Newtonian fluid across the boundary layer for a stationary vertical Porous plate in air and water in the absence of magnetic field

\section{Conclusions:}

The aim of this paper is to study the unsteady MHD heat and mass transfer free convection flow of an incompressible, viscous, electrically conducting polar fluid past a semi-infinite porous moving plate whose velocity is maintained at a constant value, and embedded in a porous medium subjected to the presence of a transverse magnetic field with heat source and thermal diffusion. The method of solution applied is Galerkin finite element. Numerical results are displayed in graphs. The variation of velocity, angular velocity, temperature and concentration profiles with various governing parameters entering into the problem. The fluids taken in this study are air and water. The following conclusions are set out:

$>$ In the absence of magnetic field, the velocity and temperature decrease in water compared with air.

$>$ The velocity distribution is lower for a Newtonian fluid as compared with a polar fluid when the viscosity ratio $\beta$ is less than 0.5 .

$>$ The angular velocity increases as the plate velocity $U_{p}$ increases both in air and water.

$>$ The velocity increases with increasing values of permeability parameter $K$ both in air and water.

$>$ In case of cooling of the plate $G \mathrm{r}>0$, the velocity decreases and angular velocity increases in water. In case of heating of the plate $G r<0$, the velocity decreases and angular velocity increases in air.

$>$ As source parameter $S$ increases, the velocity increases in air and decreases in water.

$>$ In air and water both, the velocity increases and angular velocity decreases due to increasing values of diffusion parameter $\mathrm{A}_{\mathrm{T}}$.

$>$ The temperature increases as source parameter $S$ increases.

\section{References}

Aero, E. L., Bulygin, A. N. and Kuvshinskii, E. V. (1965): Asymmetric hydromechanics, Journal of Applied Mathematics Mechanics, Vol.29 (2), pp.333-346.

Cowling, T. J. (1957): Magneto-hydrodynamics, Inter science Publishers, New York.

Cramer, K. L. and Pai, S. L. (1973): Magneto- fluid Dynamics for Engineers and Applied Physics, McGraw Hill Book Company, New York.

Dep, N. V. (1968): Equations of a fluid boundary layer with couple streses, Journal of Applied Mathematics Mech. Vol.32(4), pp.777-783. 
Eringen, A. C. (1966): Theory of micropolar fluids, Journal of Mathematics Mechanics, Vol.16, pp.1-18. http://dx.doi.org/10.1512/iumj.1967.16.16001

Eringen. A. C. (1972): Theory of thermomicropolar fluids, Journal of Mathematical Analysis and Applications, Vol.38, pp. 480-496. http://dx.doi.org/10.1016/0022-247x(72)90106-0

Ferraro, V. C. A., Plumpton, C. (1966): An introduction to Magneto Fluid Mechanics, Claranden Press, Oxford.

Gribben, R. J. (1965): The magneto-hydrodynamic boundary layer in the presence of a pressure gradient, Proc. R. Soc. London, Vol.A.287, pp.123-141.

Hassanien, I. A., Shamardan, A., Moursy, N. M. and Gorla, R. S. R. (1999): International Journal of Numerical Methods for Heat Fluid flow, Vol.9, pp.643-659.

Khandelwal, K., Gupta, A., Poonam and Jain, N. C. (2003): Effects of couple stresses on the flow through a porous medium with variable permeability in slip flow regime, Ganita,Vol.54(2), pp.203-212.

Kim, Y. J. (2000): Unsteady MHD convective heat transfer past a semi-infinite vertical porous moving plate with varivariable suction, International Journal of Engineering and Science, Vol. 38, pp. 833.

Kim, Y. J. (2001): Unsteady MHD convection flow of polar fluids past a vertical moving porous plate embedded in a porous medium, International Journal of Heat and Mass Transfer, Vol. 44, pp. 2791-2799.

Lukaszewicz. G. (1999): Micropolar Fluids-Theory and Applications, Birkhauser, Boston.

Malga, B. S. and Kishan, N. (2011): Viscous dissipation effects on unsteady free convection and mass transfer flow past an accelerated vertical porous plate with suction, Advances in Applied Science Research, Vol.2 (6), pp.460-469.

Malga, B. S. and Kishan, N. (2013): Effects of hall current on an unsteady MHD flow of heat and mass transfer along a porous flat plate with chemical reaction and viscous dissipation, International Journal of Engineering Inventions, Vol. 2(1), pp: 22-30.

Nield, D. A. and Bejan, A. (1998): Convection in porous media, 2nd edition, Springer-Verlag, Berlin.

Raptis, A. A. (1986): Flow through a porous medium in the presence of a magnetic field, International Journal of Energy Res, Vol.10, pp. 97-100. http://dx.doi.org/10.1002/er.4440100112

Raptis. A. A. and Kafousias, N. (1982): Heat transfer in flow through a porous medium bounded by an infinite vertical plate under the action of a magnetic field, International Journal of Energy Res, Vol.6, pp.241-245. http://dx.doi.org/10.1002/er.4440060305

Saxena, S. S. and Dubey, G. K. (2011): Unsteady MHD heat and mass transfer free convection flow of polar fluids past a vertical moving porous plate in a porous medium with heat generation and thermal diffusion, Advances in Applied Science Research, Vol. 2(4), pp.259-278.

Shercliff, J. A. (1965): A Text Book of Magneto-hydrodynamics, Pergamon press, London.

Singh, A. K. (2001): MHD free convection and mass transfer flow with heat source and thermal diffusion, Journal of Energy Heat and Mass Transfer, Vol.23, pp.167-178.

Soundalgekar, V. M. (1973): Free convection effects on the oscillatory flow past an infinite, vertical porous plate with constant suction, Proc. R. Soc. London, Vol. A 333, pp. 25-36.

Soundalgekar, V. M. and Takhar, H. S. (1977): MHD Oscillatory flow past a semi-infinite plate, AIAA Journal, Vol.15, pp.457-458.

Takhar, H. S. and Ram, P. C. (1994): Magnetohydrodynamic Free Convection Flow of Water at 4 Degree Centigrade, through a Porous Medium, Internal Communications of Heat Mass Transfer, Vol.21, pp.371-376.

Takhar, H. S. and Ram, R. C (1991): Free convection in hydromagnetic flow of a viscous heat generating fluid with wall temperature oscillation and Hall currents, Astrophysics and Space Science, Vol.183, pp.193-198. 